\title{
Research Paper \\ Evaluating the Anti-proliferative Effects of Nanoemulsion Containing Licorice Extract and Lavender Essential Oil on Cancer
}

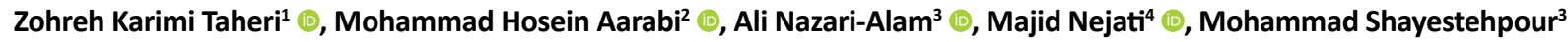 \\ (ㄷ), Hamid Reza Gilasi ${ }^{5}$ (), Afshin Salehi ${ }^{6}$ (), * Mohammad Esmaeil Shahaboddin ${ }^{1,7}$ (1)
}

1. Department of Clinical Biochemistry, Faculty of Medicine, Kashan University of Medical Sciences, Kashan, Iran.

2. Department of Clinical Biochemistry, School of Pharmacy and Pharmaceutical Sciences, Isfahan University of Medical Sciences, Isfahan, Iran

3. Department of Microbiology and Immunology, Faculty of Medicine, Kashan University of Medical Sciences, Kashan, Iran.

4. Anatomical Sciences Research Center, Kashan University of Medical Sciences, Kashan, Iran.

5. Department of Biostatistics and Epidemiology, School of Health, Kashan University of Medical Sciences, Kashan.

6. Department of Medical Laboratory Sciences, Faculty of Paramedical Sciences, Kashan University of Medical Sciences, Kashan, Iran.

7. Research Center for Biochemistry and Nutrition in Metabolic Diseases, Kashan University of Medical Sciences, Kashan, Iran.

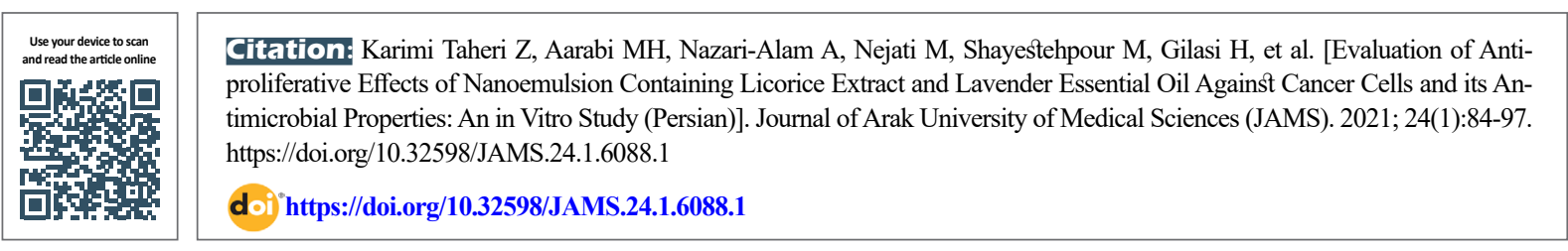

\section{(i) (3)}

Article Info:

Received: 21 Jan 2020

Accepted: $28 \mathrm{Jul} 2020$

Available Online: 01 April 2021

Keywords:

Emulsions, Lavender, Licorice, Anti-infective agents, Anticancer agent

\section{ABSTRACT}

Background and Aim Despite the anti-cancer and antimicrobial properties of licorice extract and lavender essential oil, some factors, such as low bioavailability and biodegradable, limit their therapeutic use. Using nanoparticles is a method to overcome these restrictions. This study aimed to investigate the antiproliferative effects of nanoemulsion containing licorice extract and lavender essential oil on cancer cells; we also evaluated its antimicrobial properties in vitro.

Methods \& Materials In this experimental study, nanoemulsions, containing licorice extract and lavender essential oil were developed by the spontaneous emulsion method. The anti-proliferative effect of nanoemulsion was investigated using 3-(4,5-dimethylthiazol-2-yl)-2,5-diphenyl-2H-tetrazolium bromide (MTT) colorimetric method on two cell lines HepG2 and SK-MEL-3. To measure the antimicrobial effect of 4 standard strains of Staphylococcus aureus, Escherichia coli, Pseudomonas aeruginosa, Staphylococcus epidermidis, and Minimum Inhibitory Concentration (MIC) method was used.

Ethical Considerations This study was approved by the Ethics Committee of Kashan University of Medical Sciences (Code: IR.KAUMS.MEDNT.REC.1396.106)

Results The results of MTT test on HepG2 cells indicated that the concentrations of 630, 1250, and 2500 $\mu \mathrm{g} / \mathrm{mL}$ nanoemulsions caused toxicity to the cell and led to the death of $>50 \%$ of the cells (IC50 $=401 \mu \mathrm{g} /$ $\mathrm{mL}$; $\mathrm{P}<0.05)$. Evaluating SK-MEL3 cells revealed that except for $75 \mu \mathrm{g}$ of nanoemulsion, other concentrations induced death in $>50 \%$ of the cells (IC50 $=82 \mu \mathrm{g} / \mathrm{mL} ; \mathrm{P}<0.05$ ). In addition, nanoemulsions, with antimicrobial properties, were studied in 4 strains of bacteria; the highest antimicrobial properties were observed in Staphylococcus epidermidis.

Conclusion Nanoemulsion containing licorice extract and lavender essential oil presents antimicrobial and antiproliferative effects on the two cell lines studied. The current study results indicated that the nano emulsification of lavender essential oil and licorice extract can enhance their biological impact; thus, they can be used as a drug formulation.

\section{* Corresponding Author:}

Mohammad Esmaeil Shahaboddin, PhD.

Address: Research Center for Biochemistry and Nutrition in Metabolic Diseases, Kashan University of Medical Sciences, Kashan, Iran.

Tel: +98 (31) 55540021

E-mail: shahaboddin@kaums.ac.ir 


\section{Extended Abstract}

\section{Introduction}

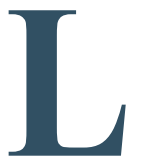

icorice and lavender plants have anticancer [4, 13] and antimicrobial [5, 15] properties; however, due to their low bioavailability and degradability, their use as a medicine has limitations $[16,17]$. One approach to overcome these restrictions is to use nanoparticles. Nanoemotions ensure the protection of biological compounds and their controlled release [19]. This study aimed to evaluate the antiproliferative effects of nanoemulsions containing licorice extract and lavender essential oil on liver cancer cell lines (HepG2) and skin cancer cells (SK-MEL3). We also explored its antimicrobial properties on Staphylococcus aureus, Escherichia coli, Pseudomonas aeruginosa, and Staphylococcus epidermidis in vitro.

\section{Materials and Methods}

In this experimental study, nanoemulsions containing licorice extract and lavender essential oil were generated by the spontaneous emulsion method. To make the nanoemulsion, the aqueous phase, consisting of glycerol, polyethylene glycol solvent, and water, as well as the oil phase, including lavender essential oil, licorice extract, and emulsifiers (Tween 20 \& Tween 80), were each prepared separately and combined and homogenized after heating. SinglePhase and transparent nanoemulsions were obtained. The antiproliferative effect of nanoemulsion on HepG2 and SKMEL3 cell lines was investigated using MTT colorimetric method. Four standard bacterial strains and the Minimum Inhibitory Concentration (MIC) method were used to measure the antimicrobial effect. The last batch of nanoemulsion, in which no growth was observed, was considered as MIC. To analyze the obtained data, a one-way Analysis of Variance (ANOVA) was employed in SPSS at $\mathrm{P}<0.05$.

\section{Results}

The results of the examination on HepG2 cells revealed that the IC50 level of nanoemulsion was $401 \mu \mathrm{g} / \mathrm{mL}$ $(\mathrm{P}<0.05)$. However, the concentration of 2500 licorice extracts could only kill $28 \%$ of the cells. The IC50 value for lavender essential oil was measured as $450(\mathrm{P}<0.05)$ (Figure 1). Examining SK-MEL3 cells suggested that the IC50 nanoemulsion concentration was $82 \mu \mathrm{g} / \mathrm{mL}(\mathrm{P}<0.05)$. Lavender essential oil had similar toxicity to nanoemulsions; however, licorice extract was toxic only at the concentrations of 1250 and $2500(\mathrm{P}<0.05)$ (Figure 2).

In the study of nanoemulsion toxicity, the desired bacterial strains were subjected to concentrations of $0.625,1.25$, $2.5,5$, and $10 \mathrm{mg} / \mathrm{mL}$ nanoemulsion (Figure 1). The collected results indicated that the nanoemulsion presented an inhibitory effect on the growth of all bacterial used strains. The highest inhibitory effect concerned Staphylococcus aureus with MIC $5 \mathrm{mg} / \mathrm{mL}$. The MIC for the other 3 strains was equal to $10 \mathrm{mg}$. Licorice extract did not affect Staphylococcus aureus and Escherichia coli but inhibited the growth of Pseudomonas and Staphylococcus epidermidis. The lavender essential oil also inhibited the growth of all strains except Pseudomonas (Table 1).

\section{Discussion and Conclusion}

The current study results revealed that the nanoemulsion is toxic to cancer cells. The anti-proliferative effect of licorice extract and lavender essential oil has been proven in previous studies $[10,20]$. When cancer cells become malignant, they produce sustained ROS, leading to tumor growth and progression [21]. Using antioxidants, such as lavender essential oil, licorice extract, or nanoemulsions prepared from them can help improve the function of anti-cancer agents by reducing the amount of ROS.

Considering the effects of licorice and lavender, it was expected that the nanoemulsion form of licorice extract and

Table 1. The MIC of nanoemulsion, lavender essential oil, and licorice extract on 4 bacterial strains

\begin{tabular}{|c|c|c|c|}
\hline \multirow{2}{*}{ Bacteria } & \multicolumn{3}{|c|}{ MIC Rate (mg / ml) } \\
\hline & Nanoemulsion & Essence & Licorice Extract \\
\hline Staphylococcus aureus & 10 & 5 & - \\
\hline Escherichia coli & 10 & 2.5 & - \\
\hline Pseudomonas aeruginosa & 10 & - & 1.25 \\
\hline Staphylococcus epidermidis & 5 & 5 & 5 \\
\hline
\end{tabular}

$(-)$ : No effect on bacterial growth. 


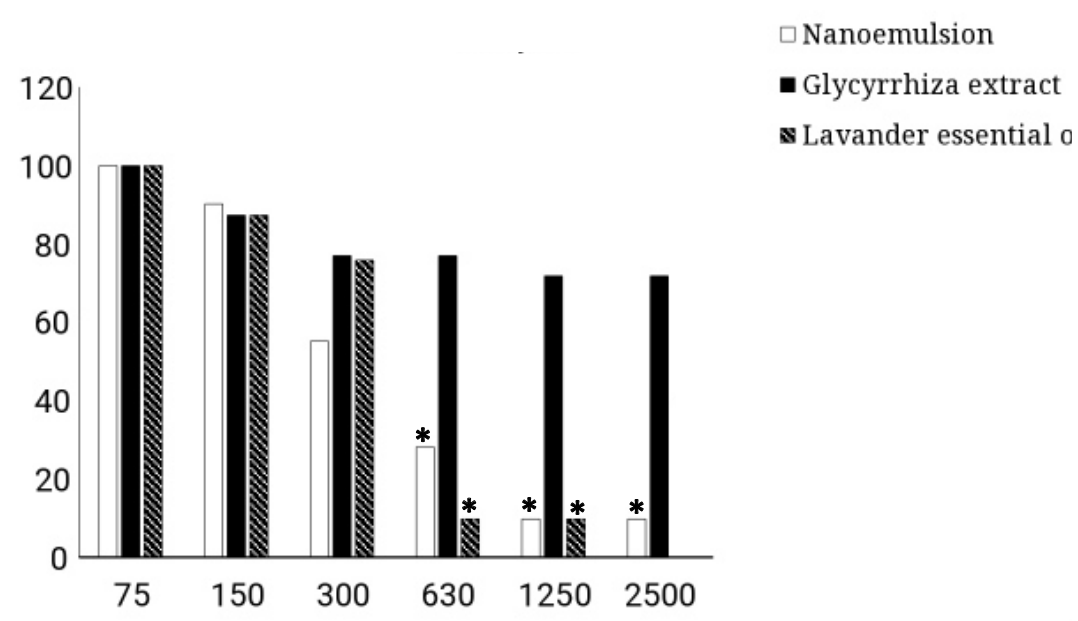

Figure 1. The percentage of HEPG2 cells surviving against different concentrations of nanoemulsion

*A significant difference of $>50 \%(\mathrm{P}<0.05)$.

lavender essential oil present a high potential to become a formulation with anti-proliferative properties against cancer cells due to its physical stability. The present study revealed that the mentioned nanoemulsion has such a property. Furthermore, the cytotoxicity of nanoemulsion is higher than licorice extract and equal to lavender essential oil. Given the hydrophobicity and volatility of the essential oils, the conversion of the essential oil into a stable nanoemulsion can be useful. These results were consistent with those of the study of Ziaee and associates [25].

The antimicrobial effects of nanoemulsion highlighted that this nanoemulsion had the highest level of inhibition on the growth of Staphylococcus epidermidis; its inhibitory effect was the same on other strains. The antibacterial effect of lavender essential oil was greater than nanoemulsion in all strains; however, licorice extract provided an inhibitory effect only on Pseudomonas and Staphylococcus epidermidis.

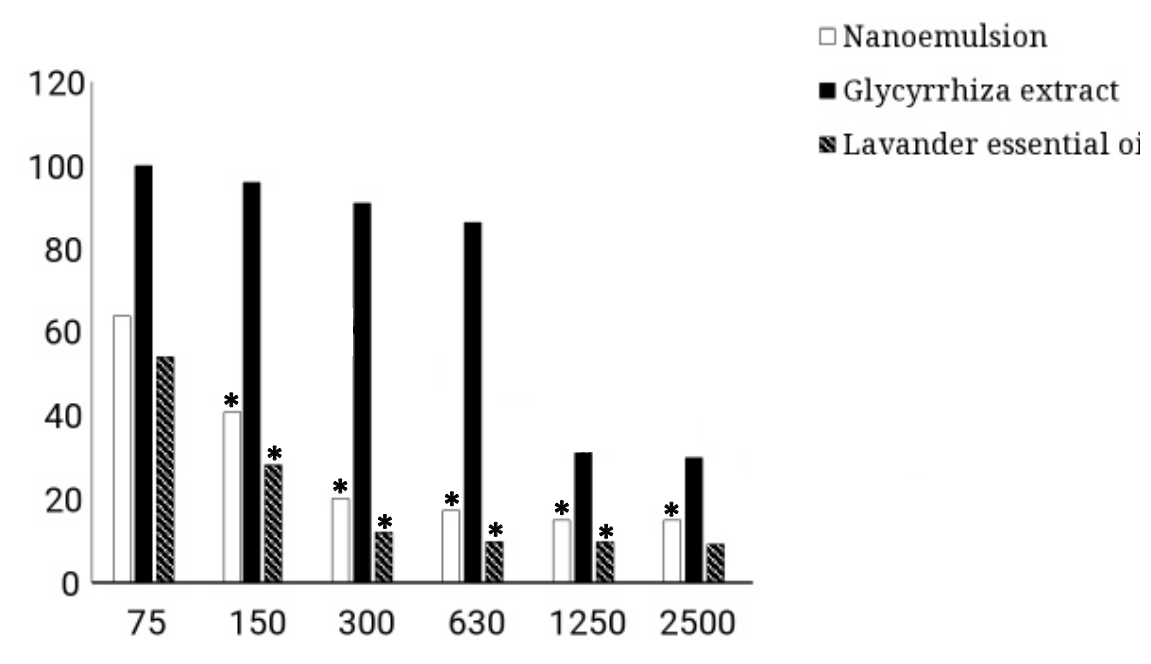

Figure 2. The survival percentage of SK-MEL3 cells against different concentrations of nanoemulsion

*A significant difference of $>50 \%(\mathrm{P}<0.05)$. 


\section{Ethical Considerations}

\section{Compliance with ethical guidelines}

This study was approved by the Research Ethics Committee of Kashan University of Medical Sciences (Code:

IR.KAUMS.MEDNT.REC.1396.106).

\section{Funding}

The paper was extracted from the MSc. thesis of the first author, Department of Clinical Biochemistry, Faculty of medicine, Kashan University of Medical Sciences (KAUMS, Grant No: 96203).

\section{Authors' contributions}

Conceptualization and validation methodology of data analysis: Mohammad Esmaeil Shahaboddin, Mohammad Hossein Aarabi; Research, analysis, and sources: Ali Nazari-Alam, Mohammad Shayestehpour, Majid Nejati, Hamidreza Gilasi, and Afshin Salehi; Drafting: Zohreh Karimi Taheri.

\section{Conflicts of interest}

The authors declared no conflicts of interest.

\section{Acknowledgements}

We would like to thank the esteemed the Vice Chancellor for Research and Technology of Kashan University of Medical Sciences. 
This Page Intentionally Left Blank 


\section{بررسى اثرات ضد تكثيرى نانوامولسيون حاوى شيرين بيان و لاواند بر عليه سلولهاى سرطانى و خواص ضد ميكروبى آن}

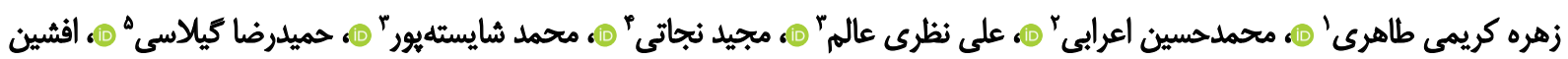

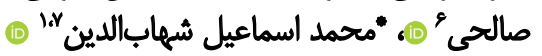

ا. ا. كروه بيوشيمى بالينى، دانشكده يزشكى، دانشكاه علوم يزشكى كاشان، كاشان، يراين.

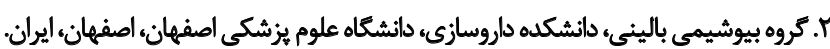

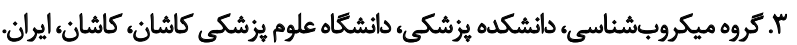

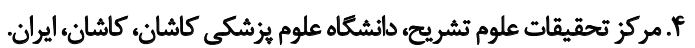

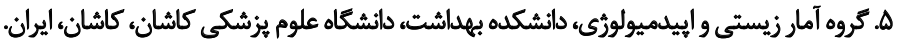

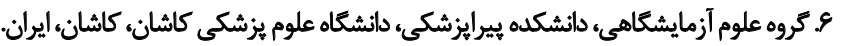

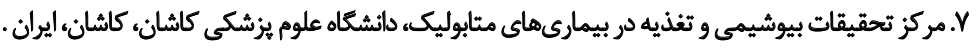

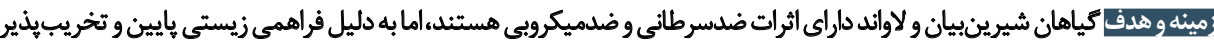

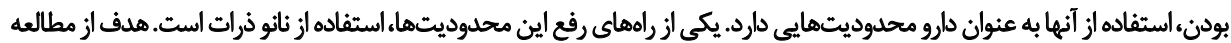

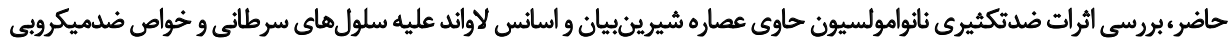

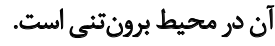

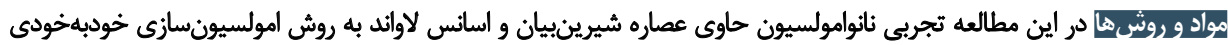

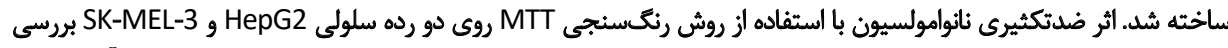

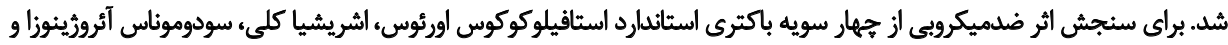

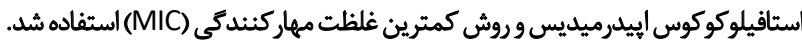

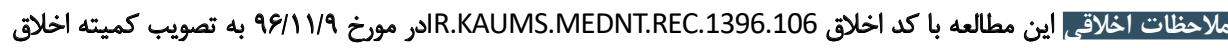

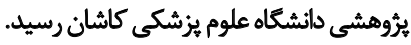

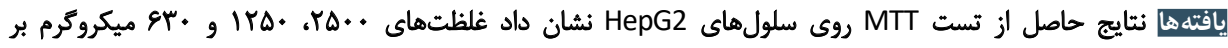

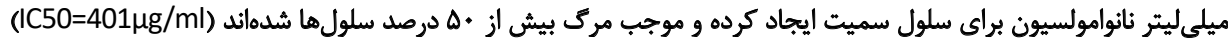

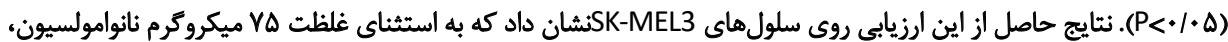

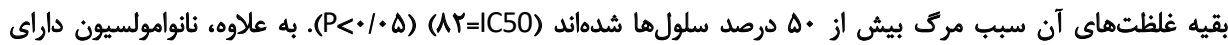

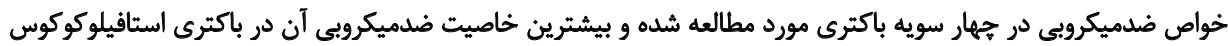

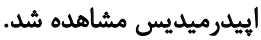

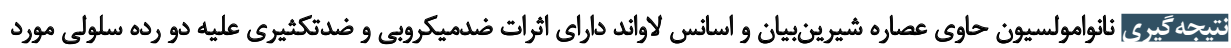

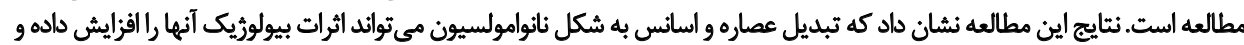
به عنوان يك فرمولاسيون دارويى جديد بن به كار رودان

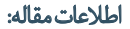

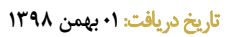
تاريخ يذيرش: V+ مرداد

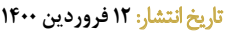

كليدوازوها:

شيرينبيان، لاوانده

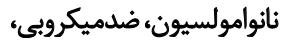

ضدتكثيرى 
كند [ [ []]. در مطالعه ديكرى نشان داده شده كه اسانس لاواند مى تواند از رشد شش كونه باكتريايى در محيط كشت جلئ جلوكيرى

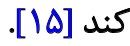

با وجود يتانسيل بالاى اين كياهان در جلوقيرى از رشد

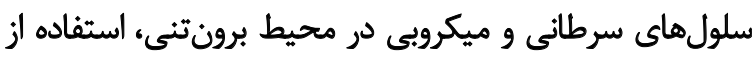

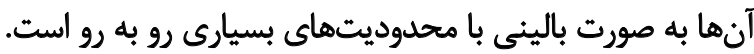

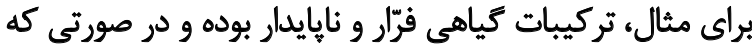

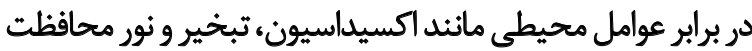

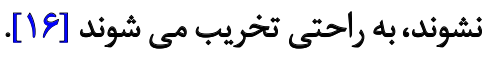

به علاوه، حلاليث يايين در آب و وزن مولكولى بالا سبب شده تا ئا

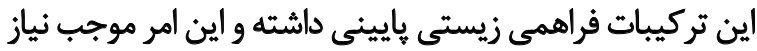

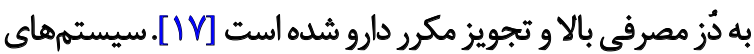

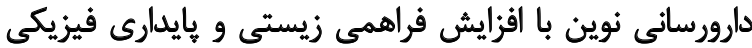

توانستهاند استفاده از اين تركيبات راكئ فارامدتر كنئد [1/1)].

يكى از اين روشهاى نوين، نانوامولسيونه كردن تركيبات

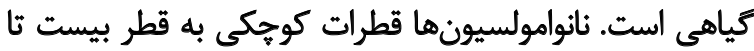

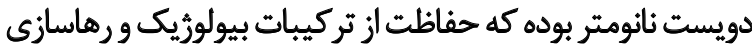

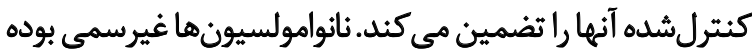

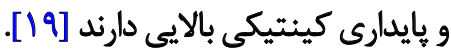

محققان نشان دادهاند كه كارايى بعضى از اسانسهاى تياهى

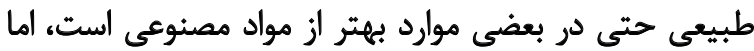

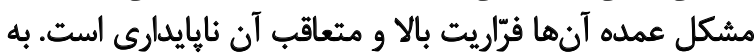

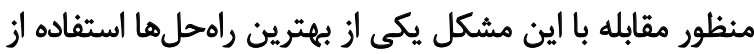

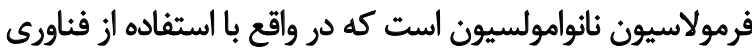

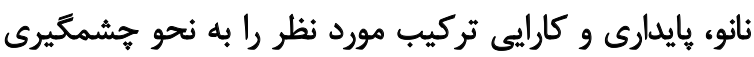

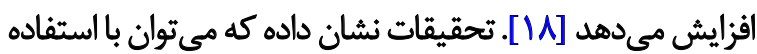

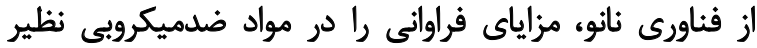

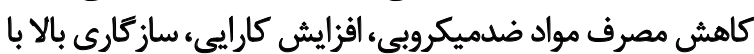

محيطزيست و بهبود كيفيت ايجاد كرد [19] كاريكي

با توجه اثرات مطلوب دو كياه شيرينبيان و لاواند و با در نظر

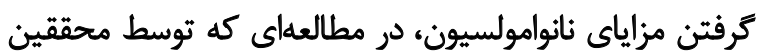

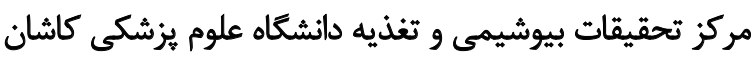

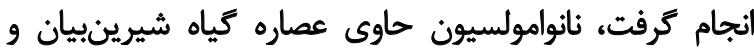

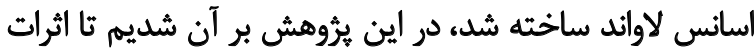

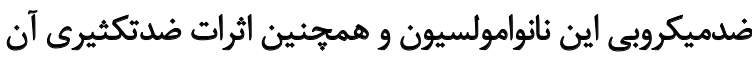
بر دو رده سلول سرطانى را بررسى كنيم.

$$
\text { مواد ورش روشها }
$$

ساخت نانوامولسيون حاوى كياهان لاواند و شيرينبيان:

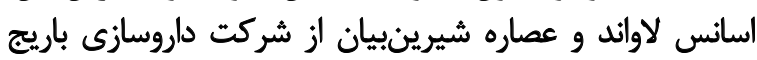
اسانس خريدارى شد. جهت ساخت نانوامولسيون از روشي الدان باري

مelod

استفادهاز كياهان براي درمان بيمارىها بيشينه تاريخى داشته

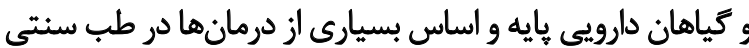

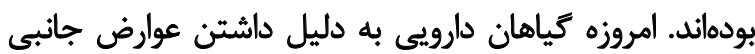

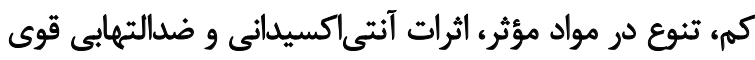

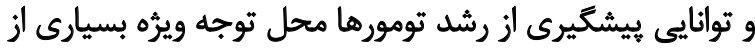

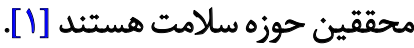

در دهاهاى اخير كياهان به عنوان يكى از منابع اصلى براي

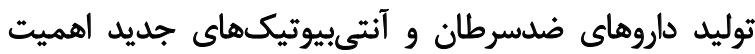

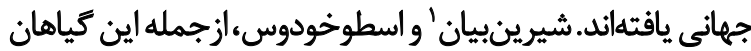

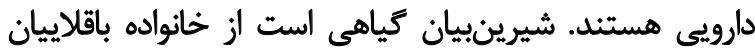

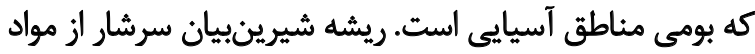

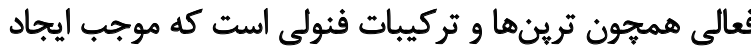

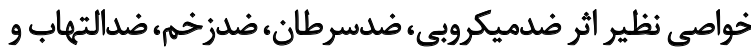

ضدحساسيت شيرين بيان مى شوند [r]

در مطالعات مختلف اثر ضدسرطانى اين گياه بررسى شده

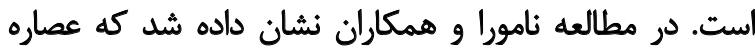

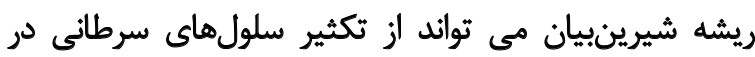

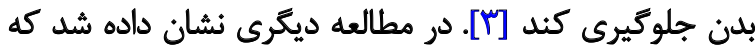

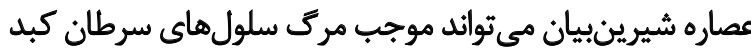

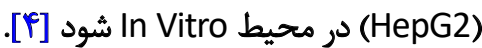
ريشه شيرينبيان اثر ضدباكترى نيز دارد.نيتاليكار و همكاران،

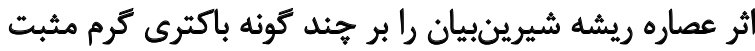

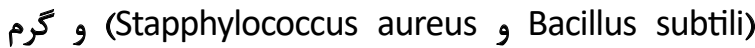

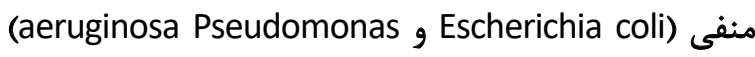
بررسى كرده و نشان دادند كه تمامى اين باكترىها به عصاره شيرينبيان حساس هستند [ه] ن.

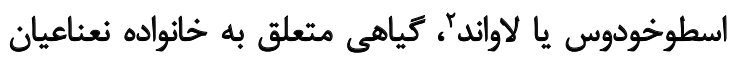

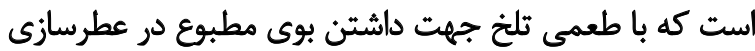

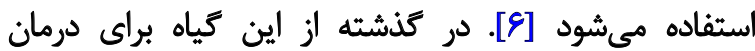

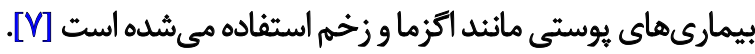

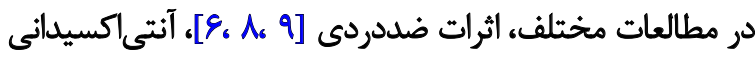

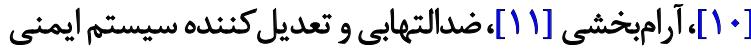

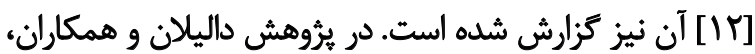

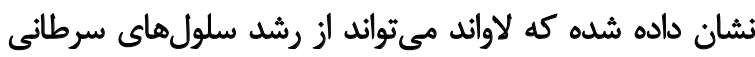
لنفوم هوجكين جلوكيرى كند [11ان]

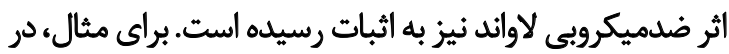

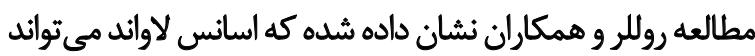
از رشد باكترىهاى مقاوم به متىسيلين (MRSA) جلوكيرى داده

1. Glycyrrhiza Glabra

2. Lavandula Angustifolia 
درمرحلهبعد،جذبنمونههابادستغاهميكرويليت ريدر (Dena،

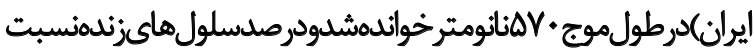
به جذب نورى سلول هاى كنترل هحاسبه شد.

تعيين اثر ضدميكروبى: به منظور تعيين حداقل غلظت

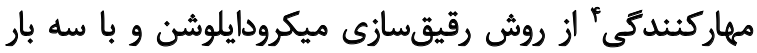

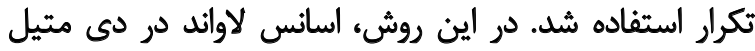
سولفوكسايد • ا درصد تهيه شد. شد.

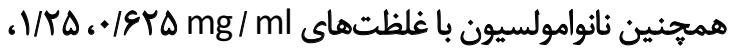

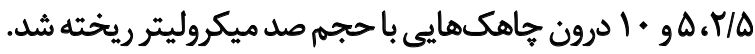

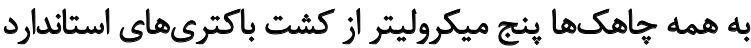

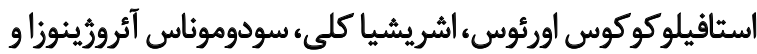
استافيلوكوكوس إيدرميديس با غلظت نيهم مك فارلند به به همراه ه9 ميكروليتر محيط مولر هينتون براث اضافه شد. شيد.

از مولر هينتون براث و سوسيانسيون باكترى به تنهايى به

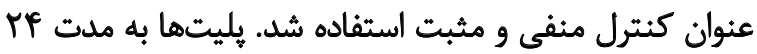

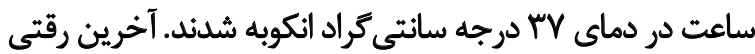

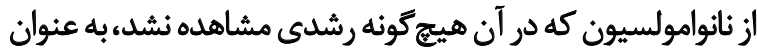
MIC

آناليز آمارى دادهها: به منظور آناليز دادهها از آزمون آناليز

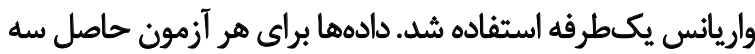

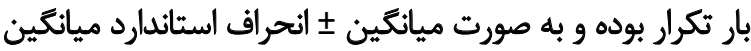

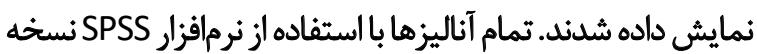

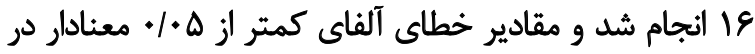
نظر كرفته شد.

batiols

سميت سلولى غلظت هاي مختلف نانوامولسيون حاوى عصاره شيرينبيان و اسانس لاواند بر سلولهاي كبدى مانى رده

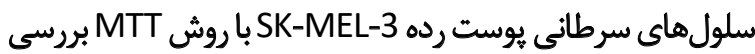

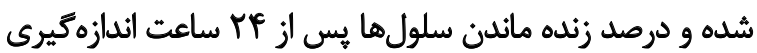

نتايج حاصل از اين ارزيابى روى سلولهاي HepG2 نشان داد

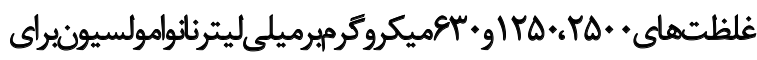

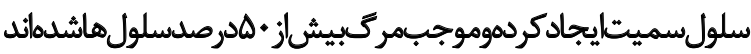
. $(\mathrm{P}<\bullet / \bullet$ ه) $(\mathrm{IC} 50=401 \mu \mathrm{g} / \mathrm{ml})$

عصاره شيرينبيان در هقايسه با نانوامولسيون سميت كمترى

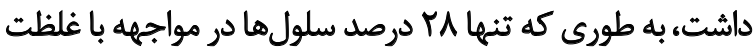
. .

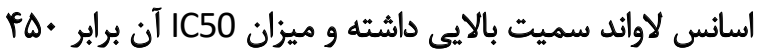

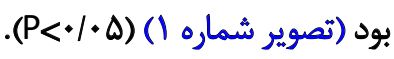

امولسيونسازى خودبيهخودى استفاده شد [19]. در مطالعه

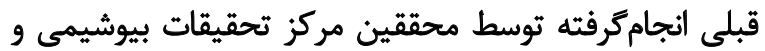

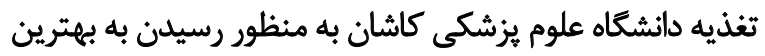

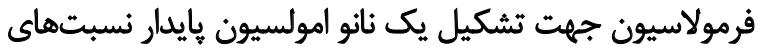

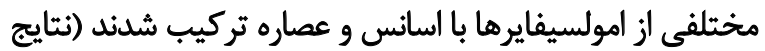
منتشر نشده است).

نتايج حاكي از آن بود كه بهترين نسبت براي امولسيفايرها

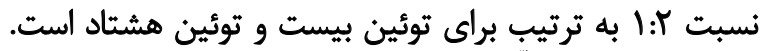

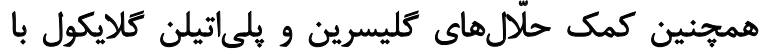
نسبت ا: الستفاده شد.

به منظور تهيه نانوامولسيون، فاز آبي شامل كمك حآلال ها و

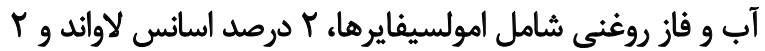

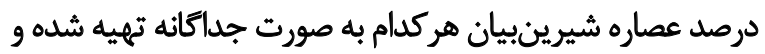

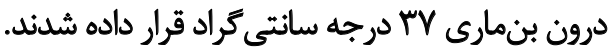

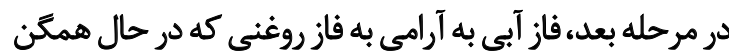

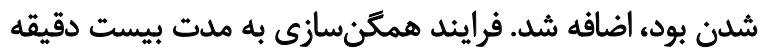

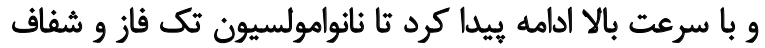

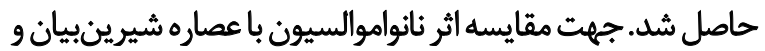

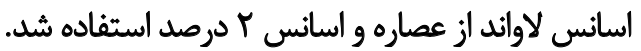
تعيين اثر ضدتكثيرى نانوامولسيون عليه سلول هاي سرطانى:

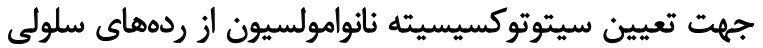
سرطان كبد (HepG2) و سلول سرطائي يوستيت (SK-MEL3)

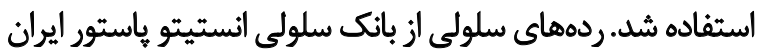
خريدارى شد.

به منظور بررسي سميت سلولى از روش MTT استفاده شد.

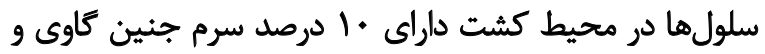

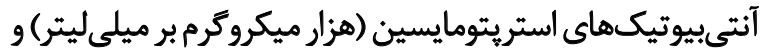
ينى سيلين (صد واحد بر ميلى ليتر) كشت داده شدند. محيط دهاي

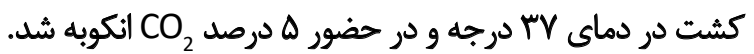

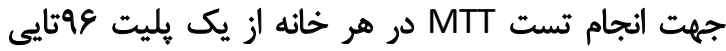

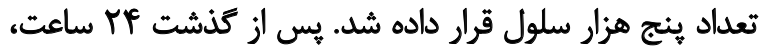

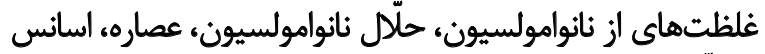

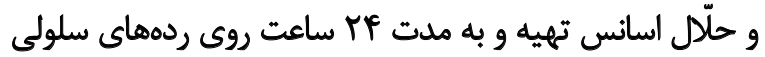
تيمار شد.

يس از كُدشت زمان فوق، محتواي جاهك ها به دقت خارج شده

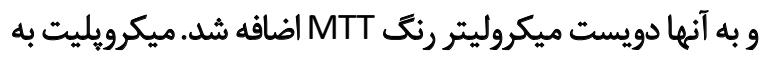

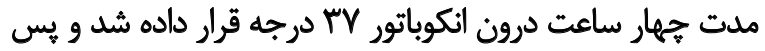

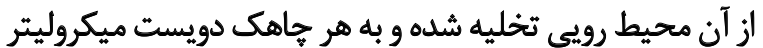
محلول DMSO D D اضافه شد. 


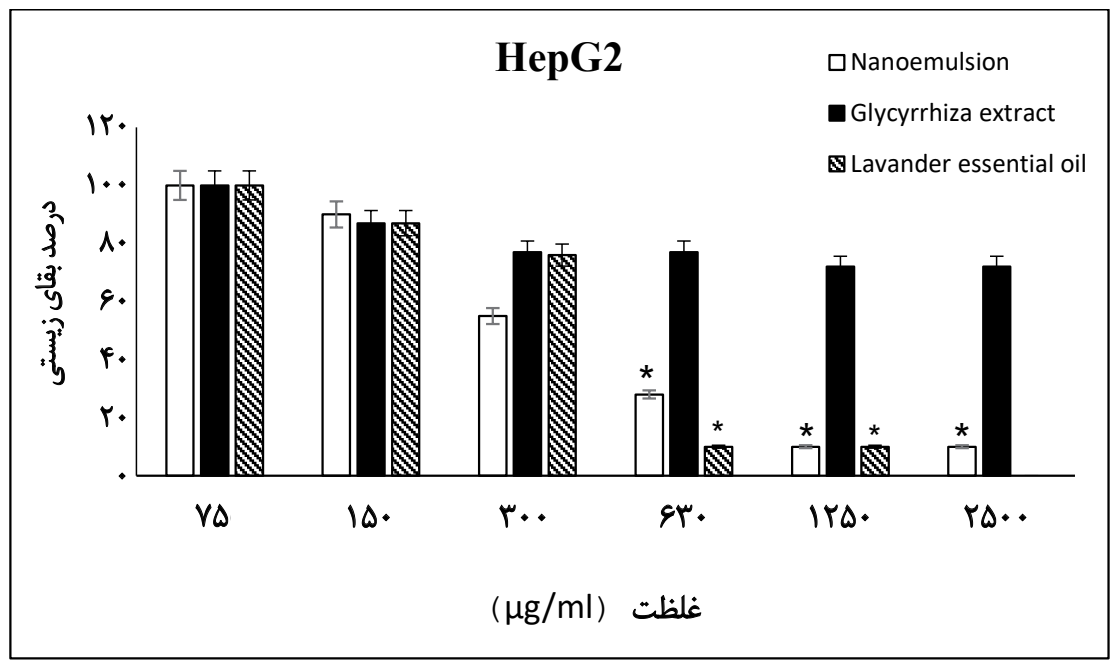

تصوير ا. درصد بقاى سلولهاى HEPG2 در برابر غلظتهاى مختلف نانوامولسيون

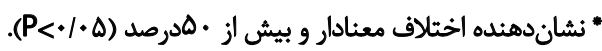

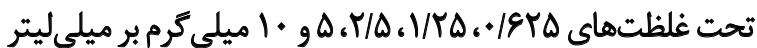

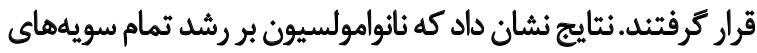

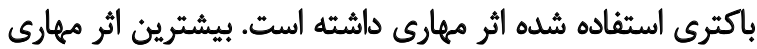

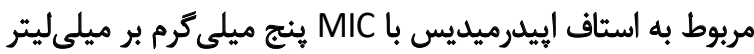

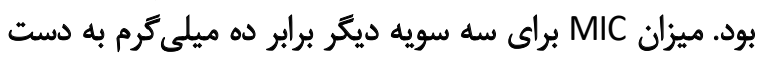

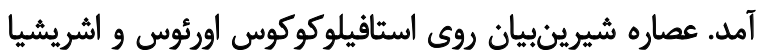

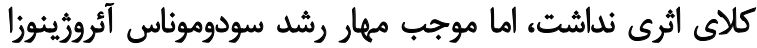

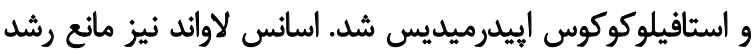

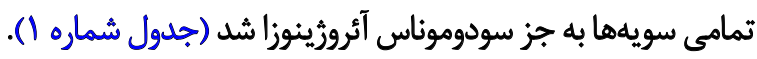
ث هدف از اين مطالعه بررسى اثر ضدميكروبى نانوامولسيون
نتايج حاصل از تست MTT روى سلولهاي

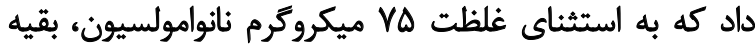

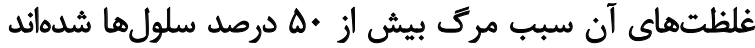
. (P< $1 \cdot \Delta)(I C 50=\wedge r)$

اسانس لاواند نيز سميتى مشابه نانوامولسيون ايجاد كرده بود،

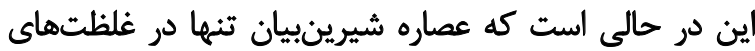

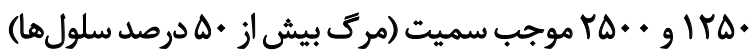

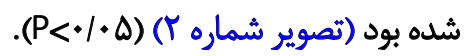

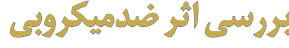

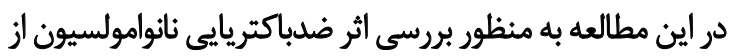

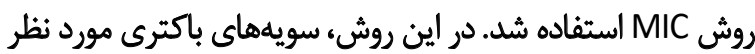

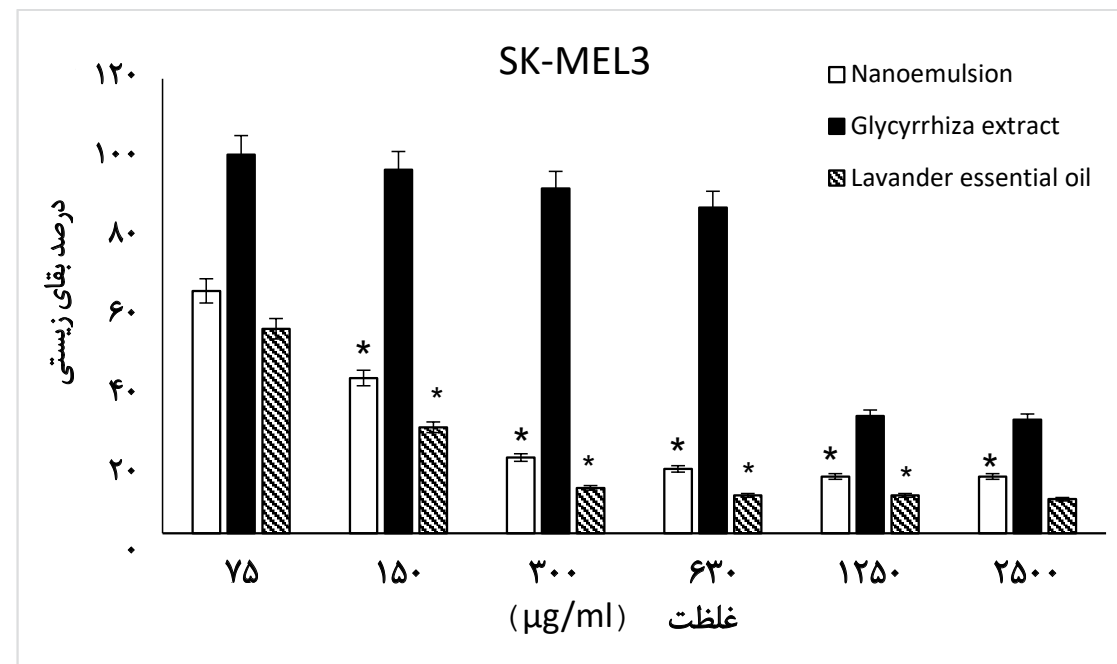

تصوير T. درصد بقاى سلولهاي SK-MEL3 برابر غلظتهاي مختلف نانوامولسيون * نشاندهنده اختلاف معنادار و بيش از • هدرصد (ه (P<). 
جدول ا. ميزان CIM نانوامولسيون، اسانس لاواند و عصاره شيرينبيان بر جهار سويه باكترى

\begin{tabular}{|c|c|c|c|}
\hline \multicolumn{3}{|c|}{ ميزان MIC (mg / ml) } & \multirow{2}{*}{ باكترى } \\
\hline عصاره شيرينبيان & السانس & نانوامولوسيون & \\
\hline- & $\Delta$ & 1. & اسثافيلوكوكوس اورئوس \\
\hline- & $r / \Delta$ & 1. & اشريشيا كلاي \\
\hline I/TA & - & 1. & سودوموناس آئروزينوزا \\
\hline$\Delta$ & $\Delta$ & $\Delta$ & استافيلوكوكوس اييلرميديس \\
\hline
\end{tabular}

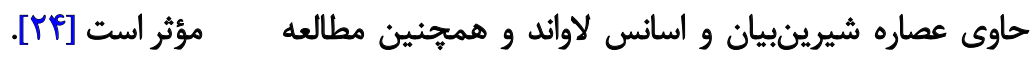

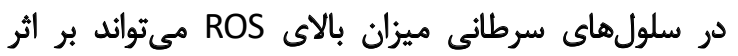

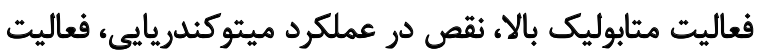

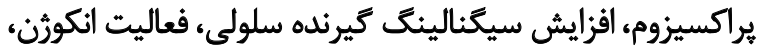

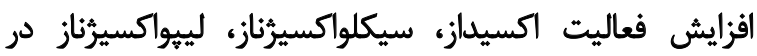

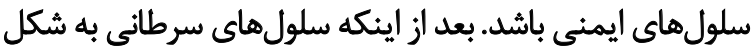

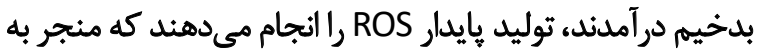

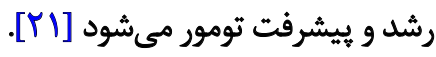

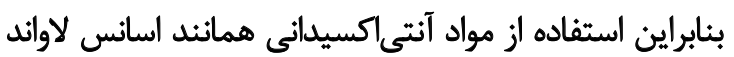

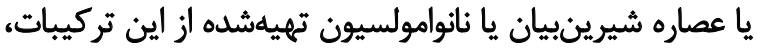

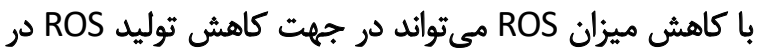

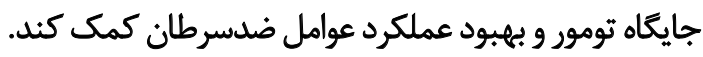

با توجه به اثرات و خواص يادشده از كياه شيرينبيان و لاواند،

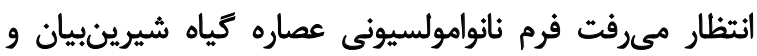

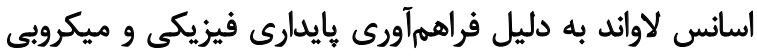

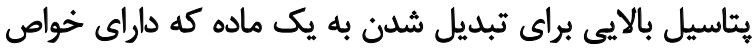

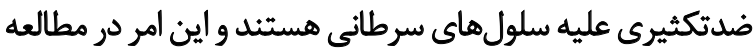

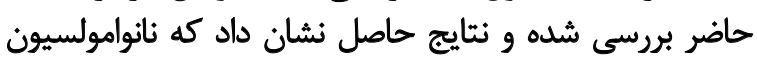
يادشده داراى جنين خاصيتى است.

همجنين، در مقايسه اثر سميت سلولى نانوامولسيون با اسانس نانس

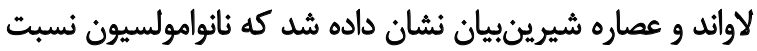

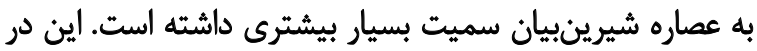

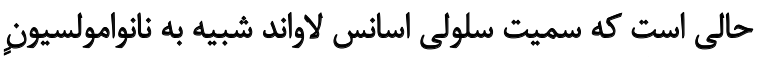

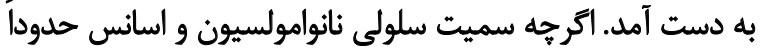

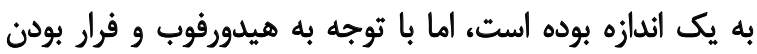

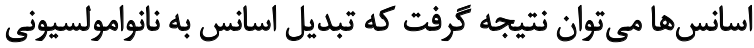

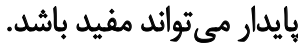

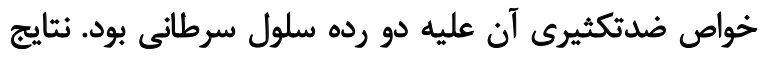

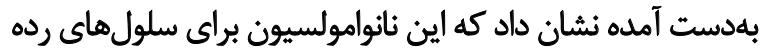

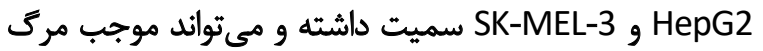
سلول هاي سرطانى شود. مطالعات متعددى در زميئه سميت سلولى و اثر ضدسرطانى

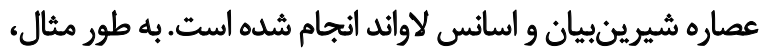

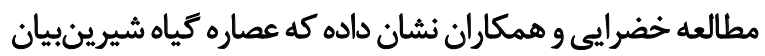

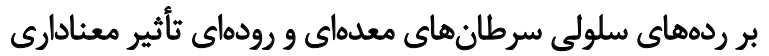

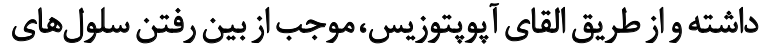

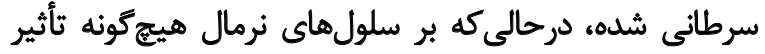

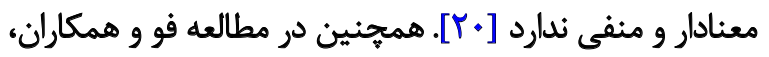

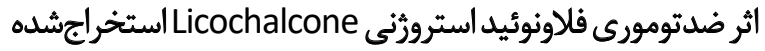

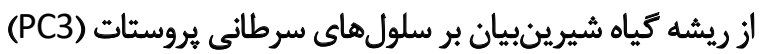

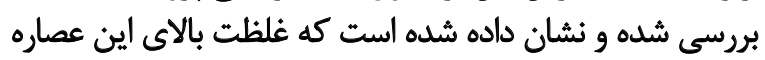
براى سلولهاى سرطان يستان سميت دارد [ [Y]". دريثروهش ديكرى بيان شد كه غلظت ده ميكروكرم شيرينبيان

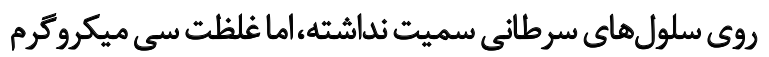

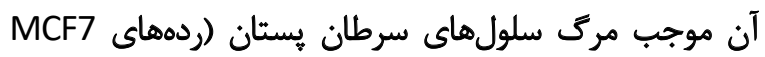

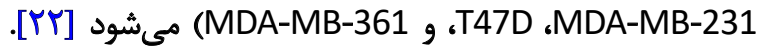

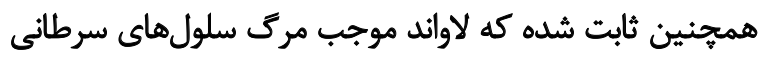

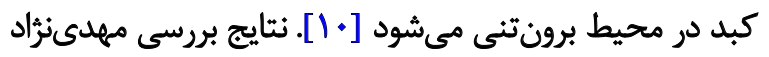

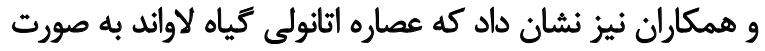

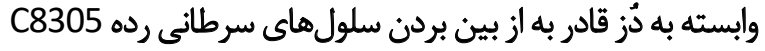

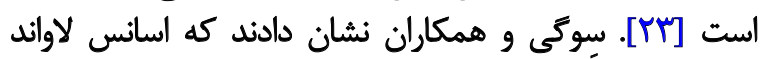

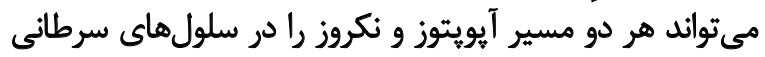

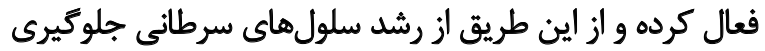

در مطالعه ضيايى و همكاران در بررسى تأثير اسانس و

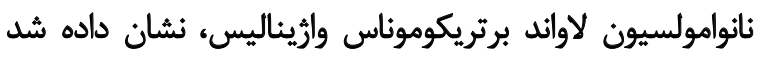

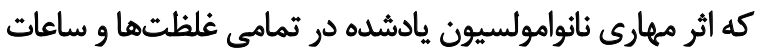

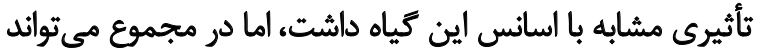

به علاوه، در اين يُروهش بيان شده است كه اثر مهارى اسانس

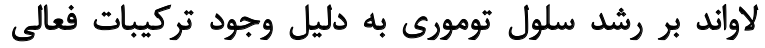

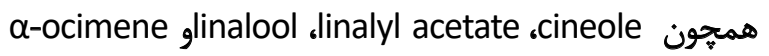


مئشوند. از سوى ديكر اين تركيبات ميتوانند موجب اختلال

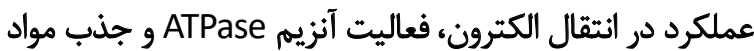

مغذى شوند [Y\%، YV]

بنابراين با توجه به اين كه آزمون ضدميكروبى انجامشده در

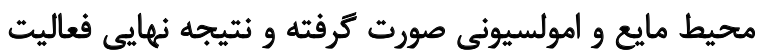

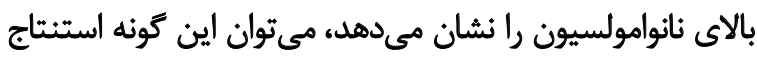

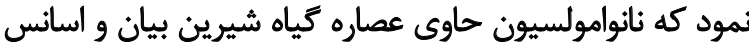

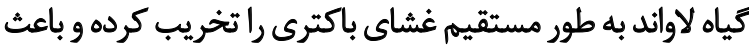

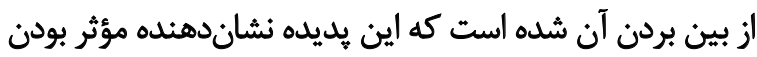

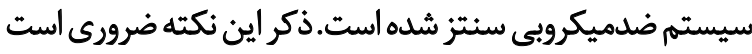
كه بررسى مكانسيم دقيق عملكرد ضدميكروبى نانيق نانوامولسيون

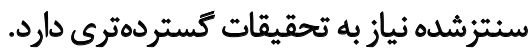

\section{نتيجلئيرى}

نتايج مطالعه حاضر نشان داد كه نانوامولسيون حاوى عصاره

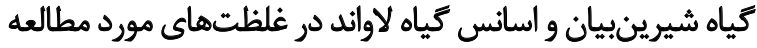

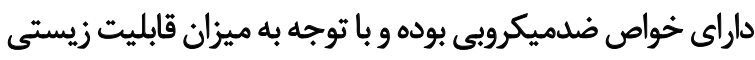

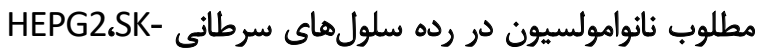

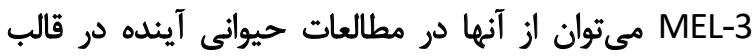
فرمولاسيونهاي دارويى استفاده كرد.

\section{مالاحظاث اخلاقى}

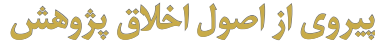

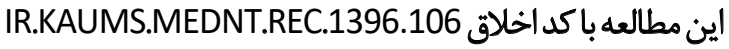

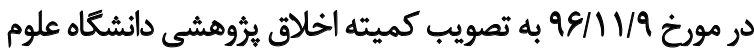

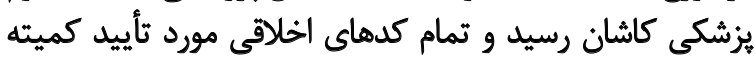

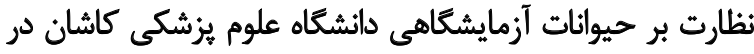
طول انجام آزمايشات رعايت شدات آنمايش

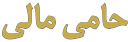

نتايج اين تحقيق حاصل بايانانامه كارشناسى ارشد نويسنده

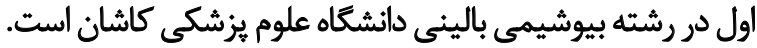

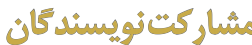

مفهومسازى و روششناسى اعتبارسنجى تحليل دادها:

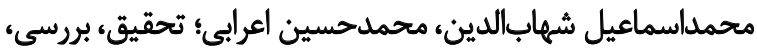

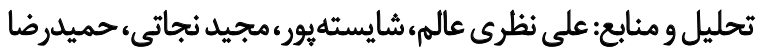

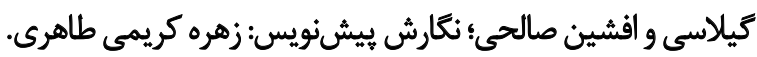

انتخاب مطلوبى به عنوان مهاركننده رشد بر تريكوموناس واريناليس باشد [Yه]]. در مطالعه ما نيز اسانس و نانوانوامولسيون

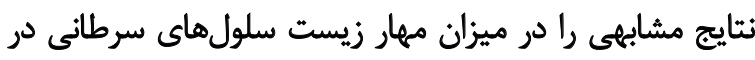
ردهاي سلولى مورد مطالعه داشتئد.

در بخش دوم اين يرؤهش، اثر ضدميكروبى نانوامولسيون

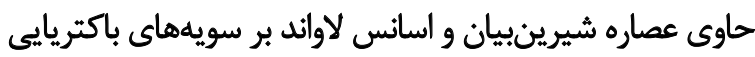

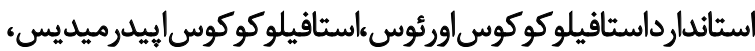

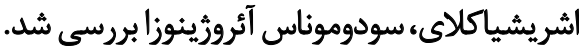

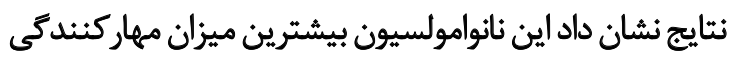

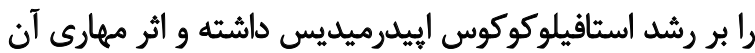

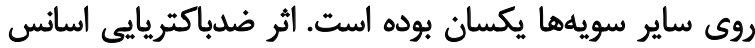

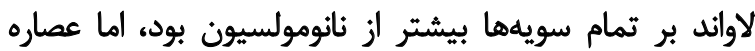

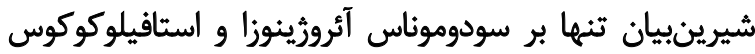

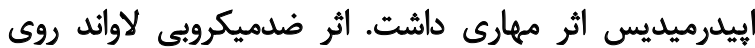

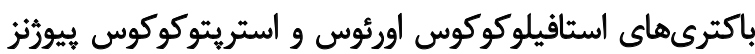

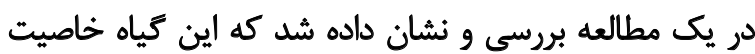

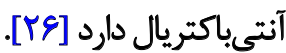

در مطالعه معصومى و همكاران در بررسى اثرات ضدميكروبى إنى إني

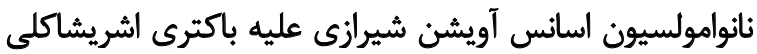

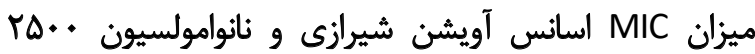

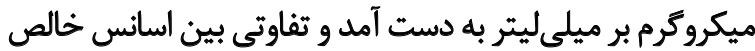

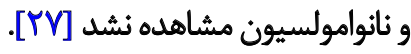

تنتايج حاصل از اين بررسى با مطالعه حاضر يكسان بود، با اين

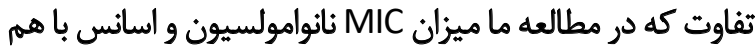

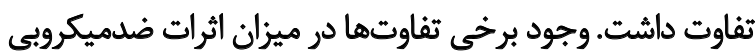

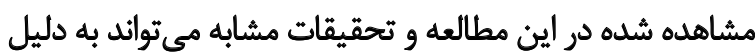

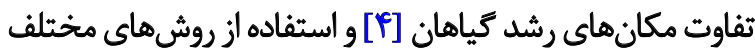

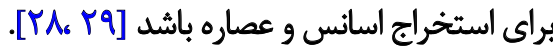
با وجود اين، اثرات ضدميكروبى سويدهاي مختلف اين تياهان

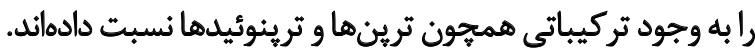

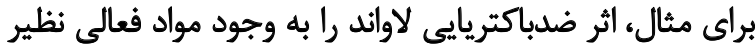

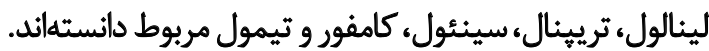

ثابت شده است اين تركيبات مىتوانند با مختل كردن كار

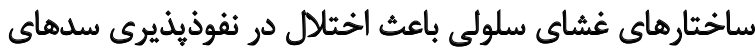

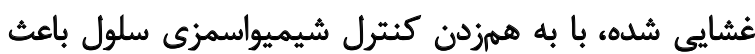

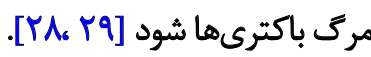

تحقيقات در مورد مكانيسم عمل تركيبات اسانس هاى حاوى

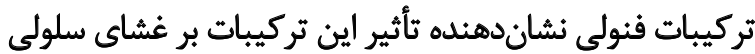

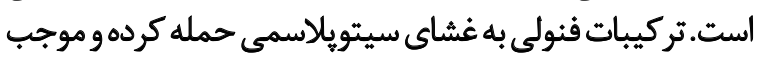

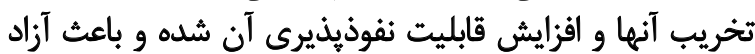

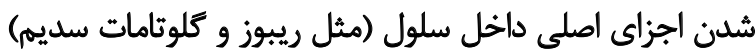




$$
\text { تُعاوض منأقع }
$$

نويسندكان اعلام مي كنين كه هيجكونه تضاد منافعى در

خصوص يرؤهش حاضر وجود ندارد.

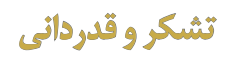

ازمعاونت محترم تحقيقات وفناورى دانشكاه علوم يزشكى كاشان

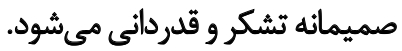




\section{Reference}

[1] Bent S. Herbal medicine in the United States: Review of efficacy, safety, and regulation. J Gen Intern Med. 2008; 23(6):854-9. [DOI:10.1007/ s11606-008-0632-y] [PMID] [PMCID]

[2] Hosseinzadeh H, Nassiri-AsI M. Pharmacological effects of Glycyrrhiza spp. and its bioactive constituents: Update and review. Phytother Res. 2015; 29(12):1868-86. [DOI:10.1002/ptr.5487] [PMID]

[3] Nomura T, Fukai T, Akiyama T. Chemistry of phenolic compounds of licorice (Glycyrrhiza species) and their estrogenic and cytotoxic activities. Pure Appl Chem. 2002; 74(7):1199-206. [DOI:10.1351/ pac200274071199]

[4] Basar N, Oridupa OA, Ritchie KJ, Nahar L, Osman NMM, Stafford A, et al. Comparative cytotoxicity of Glycyrrhiza glabra roots from different geographical origins against immortal human keratinocyte ( $\mathrm{HaCaT}$ ), lung adenocarcinoma (A549) and liver carcinoma (HepG2) cells. Phytother Res. 2015; 29(6):944-8. [DOI:10.1002/ptr.5329] [PMID]

[5] Nitalikar MM, Munde KC, Dhore BV, Shikalgar SN. Studies of antibacterial activities of Glycyrrhiza glabra root extract. Int J Pharm Tech Res. 2010; 2(1):899-901.

[6] Ghadiri MK, Gorji A. Lavender for medicine: A brief review of clinical effects. Avicenna. 2002; 1:23-7. http://citeseerx.ist.psu.edu/viewdoc/ download?doi=10.1.1.116.6864\&rep=rep1\&type=pdf\#page=24

[7] Shiina Y, Funabashi N, Lee K, Toyoda T, Sekine T, Honjo S, et al. Relaxation effects of lavender aromatherapy improve coronary flow velocity reserve in healthy men evaluated by transthoracic Doppler echocardiography. Int J Cardiol. 2008; 129(2):193-7. [DOI:10.1016/j. ijcard.2007.06.064] [PMID]

[8] Shahnazi M, Nikjoo R, Yavarikia P, Mohammad-Alizadeh-Charandabi S. Inhaled lavender effect on anxiety and pain caused from intrauterine device insertion. J Caring Sci. 2012; 1(4):255. [doi: 10.5681/ jcs.2012.035

[9] SILVA GL, Luft C, Lunardelli A, Amaral RH, MELO DA, Donadio MV, et al. Antioxidant, analgesic and anti-inflammatory effects of lavender essential oil. An Acad Bras Cienc. 2015; 87(2 S):1397-408. [DOI:10.1590/0001-3765201520150056] [PMID]

[10] Kozics K, Srancikova A, Sedlackova E, Horvathova E, Melusova M, Melus $\mathrm{V}$, et al. Antioxidant potential of essential oil from Lavandula angustifolia in in vitro and ex vivo cultured liver cells. Neoplasma. 2017; 64(4):485-93. [DOI:10.4149/neo_2017_401] [PMID]

[11] Prusinowska R, Śmigielski KB. Composition, biological properties and therapeutic effects of lavender (Lavandula angustifolia L). A review. Herba Pol. 2014; 60(2):56-66. [DOI:10.2478/hepo-2014-0010]

[12] Imre S, Eşianu S, Miklos A, Tiuca I, Dicher I, Tero-Vescan A, et al. Qualitative assay of essential oils of Lavender and Peppermint in commercial products through spectral and chromatographic methods. Farmacia. 2016; 64(6):857-62. https://farmaciajournal.com/wpcontent/uploads/2016-06-art-09-Imre_857-862.pdf

[13] Dalilan S, Rezaei-Tavirani M, Nabiuni M, Heidari-Keshel S, Azodi $\mathrm{MZ}$, Zali $\mathrm{H}$. Aqueous extract of Lavender angustifolia inhibits lymphocytes proliferation of Hodgkin's lymphoma patients. Iran J Cancer Prev. 2013; 6(4):201-8. [PMCID]

[14] Roller S, Ernest N, Buckle J. The antimicrobial activity of high-necrodane and other lavender oils on methicillin-sensitive and-resistant Staphylococcus aureus (MSSA and MRSA). J Altern Complement Med. 2009; 15(3):275-9. [DOI:10.1089/acm.2008.0268] [PMID]
[15] Hossain S, Heo H, De Silva B, Wimalasena S, Pathirana H, Heo G-J. Antibacterial activity of essential oil from lavender (Lavandula angustifolia) against pet turtle-borne pathogenic bacteria. Lab Anim Res. 2017; 33(3):195-201. [DOI:10.5625/lar.2017.33.3.195] [PMID] [PMCID]

[16] Bakkali F, Averbeck S, Averbeck D, Idaomar M. Biological effects of essential oils-a review. Food Chem Toxicol. 2008; 46(2):446-75 [DOI:10.1016/j.fct.2007.09.106] [PMID]

[17] Raut JS, Karuppayil SM. A status review on the medicinal properties of essential oils. Ind Crops Prod. 2014; 62:250-64. [DOI:10.1016/j. indcrop.2014.05.055]

[18] Thakkar PJ, Madan P, Lin S. Transdermal delivery of diclofenac using water-in-oil microemulsion: Formulation and mechanistic approach of drug skin permeation. Pharm Dev Technol. 2014; 19(3):373-84. [DO l:10.3109/10837450.2013.788658] [PMID]

[19] El Asbahani A, Miladi K, Badri W, Sala M, Addi EA, Casabianca H et al. Essential oils: From extraction to encapsulation. Int J Pharm 2015; 483(1-2):220-43. [DOI:10.1016/j.jpharm.2014.12.069] [PMID]

[20] Khazraei-Moradian S, Ganjalikhani-Hakemi M, Andalib A, Yazdani $R$, Arasteh J, Kardar GA. The effect of licorice protein fractions on proliferation and apoptosis of gastrointestinal cancer cell lines. Nutr Cancer. 2017; 69(2):330-9. [DOI:10.1080/01635581.2017.1263347] [PMID]

[21] Fu Y, Chen J, Li Y-J, Zheng Y-F, Li P. Antioxidant and anti-inflammatory activities of six flavonoids separated from licorice. Food Chem. 2013 141(2):1063-71. [DOI:10.1016/j.foodchem.2013.03.089] [PMID]

[22] Vlaisavljević S, Šibul F, Sinka I, Zupko I, Ocsovszki I, Jovanović-Šanta S. Chemical composition, antioxidant and anticancer activity of licorice from Fruska Gora locality. Ind Crops Prod. 2018; 112:217-24. [DOI:10.1016/j.indcrop.2017.11.050]

[23] Mehdinezhad Doghikolayi S, Mohammadi M. [The antioxidant and cytotoxic effects of lavandulla anguostifouliya on the $8305 \mathrm{C}$ cell line (Persian)]. New Cell Mol Biotechnol J. 2018; 8(32):91-8. https://ncmbjpiau.ir/browse.php?a_id=1146\&sid=1\&slc_lang=en

[24] Gezici S. Promising anticancer activity of lavender (Lavandula angustifolia Mill.) essential oil through induction of both apoptosis and necrosis. Ann Phytomed. 2018; 7(2):38-45. [DOI:10.21276/ ap.2018.7.2.5]

[25] Ziaei Hezarjaribi $H$, Nadeali $N$, Saeedi $M$, Soosaraei M, Jorjan ON, Momeni Z, et al. [The effect of lavender essential oil and nanoemulsion on Trichomonas vaginalis in vitro (Persian)]. Feyz. 2017 21(4):326-34. http://feyz.kaums.ac.ir/article-1-3154-en.html

[26] Giovannini D, Gismondi A, Basso A, Canuti L, Braglia R, Canini A et al. Lavandula angustifolia mll. Essential oil exerts antibacteria and anti-inflammatory effect in macrophage mediated immune response to staphylococcus aureus. Immunol Invest. 2016; 45(1):11 28. [DOI:10.3109/08820139.2015.1085392] [PMID]

[27] Masoomi VO, Tajik H, Moradi M, Forough M, Shahabi N. [Antimicrobial effects of Zataria multiflora boiss. Essential oil nanoemulsion against Escherichia coli 0157: H7 (Persian)]. Stud Med Sci. 2016 27(7):608-17. http://umj.umsu.ac.ir/browse.php?a_code=A-10-2520 $1 \&$ sid $=1 \&$ slc $\_$lang $=$en

[28] Sabbioni C, Ferranti A, Bugamelli F, Forti GC, Raggi MA. Simultaneous HPLC analysis, with isocratic elution, of glycyrrhizin and glycyrrhetic acid in liquorice roots and confectionery products. Phytochem Anal. 2006; 17(1):25-31. [DOI:10.1002/pca.877] [PMID] [PMCID] 
[29] Bradley PR. British herbal compendium: A handbook of scientific information on widely used plant drugs. United Kingdom: British Herbal Medicine Association; 1992. https://books.google.com/books/ about/British_Herbal_Compendium.html?id=IrUFBAAACAA 